CDD: 160

\title{
ONE REASON, SEVERAL LOGICS
}

\author{
EVANDRO AGAZZI \\ Department of Philosophy \\ University of Genoa \\ ITALY \\ Universidad Autónoma Metropolitana \\ Cuajimalpa \\ $M E X I C O$
}

evandro.agazzi@gmail.com

\begin{abstract}
Humans have used arguments for defending or refuting statements long before the creation of logic as a specialized discipline. This can be interpreted as the fact that an intuitive notion of "logical consequence" or a psychic disposition to articulate reasoning according to this pattern is present in common sense, and logic simply aims at describing and codifying the features of this spontaneous capacity of human reason. It is well known, however, that several arguments easily accepted by common sense are actually "logical fallacies", and this indicates that logic is not just a descriptive, but also a prescriptive or normative enterprise, in which the notion of logical consequence is defined in a precise way and then certain rules are established in order to maintain the discourse in keeping with this notion. Yet in the justification of the correctness and adequacy of these rules commonsense reasoning must necessarily be used, and in such a way its foundational role is recognized. Moreover, it remains also true that several branches and forms of logic have been elaborated precisely in order to reflect the structural features of correct argument used in different fields of human reasoning and yet insufficiently mirrored by the most familiar logical formalisms.
\end{abstract}

Keywords: Logical consequence. Psychology of logic. Descriptive and normative in logic. Commonsense reasoning.

\section{LOGIC, A POLYSEMIC CONCEPT}

A preliminary clarification, when one wants to investigate the issue of the plurality of logics, consists in distinguishing at least two meanings of this plurality. The first could be considered as the consequence of the polysemic nature of the concept logic, that is, of the fact that 
this concept is often applied to different semantic domains, and this normally appears from the fact that it is spoken of "the logic of...". For instance, we find in our language expressions such as: "the logic of the events brought about that ...", "the logic of power does not permit...", "the logic of economy, or of profit, entails that...", "the logic of research", "the logic of thought, or of discourse", and so on. It is very spontaneous to say that the "proper" use of our term is that indicated as "logic of thought or discourse", since thought (and more precisely thought explicitly expressed in a discourse) is the traditional subject matter of the specific discipline historically called logic (as the definitions of the subject matter, and even the titles of the treatises devoted to this discipline often declare ${ }^{1}$ ). One should refrain, however, from rejecting the other uses as spurious, confused, or even meaningless: they share a particular meaning of "logic", that could be indicated as the intrinsic nature, or the specific objective structure, of a given domain, from which the functioning of this domain, or the actions that are characteristic of it, can be more or less explicitly expressed by means of certain laws or rules. Therefore, we can safely decide that, when speaking of logic, we consciously intend to speak of a discipline that studies thought expressed in a discourse, and this determines its "domain". It must be admitted, however, that this delimitation is still too broad, for thought and discourse can be studied from several points of view (such as those of linguistic, grammar, etc.) that we do not consider as belonging to logic, at least today.

Indeed we normally think of logic as being concerned with that particular aspect or part of thought that consists in "reasoning" or in

${ }^{1}$ Let us only mention two titles, belonging to different historical times, and also to rather different conceptions of logic: La logique ou l'art de penser (1662) published by A. Arnaud and P. Nicole (the famous "logic of PortRoyal"): and An investigation of the laws of thought, on which are founded the mathematical theories of logic and probability, published in 1854 by G. Boole. 
constructing "arguments" (such that, even if other aspects or parts of thought are taken into consideration in logic, they are investigated only to the extent that they are relevant to the construction and evaluation of arguments). In conclusion, we can say that the domain of logic is the study of correct reasoning.. In the literature it is often spoken of valid arguments, meaning that they are formally correct, and then it is added that they must also be sound, hinting by that at certain additional requirements they must satisfy (cf. for example Haak (1978)). We use correct as an adjective denoting at the same time validity and soundness. Let us also add that, not being interested in historical reconstructions, we ignore several facts: for example, that what we consider the content of logic was called "dialectic" during several centuries, that according to other traditions (e.g. in Kant) logic contained two parts, "analytic" and "dialectic", that in the Scholastic tradition it was customary to speak of a logica maior which encompassed a variety of issues that we include today rather in epistemology and philosophy of language, and a logica minor that was much similar to the formal logic as we conceive it.

But what is reasoning? From a descriptive point of view we can say that reasoning is a particular connexion of propositions or sentences (we speak of propositions when we consider thought, and of sentences when we consider the linguistic expression of thought). ${ }^{2}$ Now we speak, for example, of an "eloquent" reasoning, of a "persuasive" resoning, of a "correct" reasoning. In the first case we mean a reasoning having an emotional impact, in the second a reasoning capable of producing other people's assent, especially when action is concerned. What do

\footnotetext{
${ }^{2}$ We are aware of the non-univocal meaning attributed by different authors to the terms "proposition", "sentence", and "statement", and to the mutual relations existing among them, especially in the discussions regarding the "truth-bearers" (cf. Haak 1978, pp. 74-85). We do not need, however, to enter this controversy and, for the sake of this paper, the distinction we have proposed will be sufficient.
} 
we mean by a "correct" reasoning? A very plausible answer is that a reasoning is correct when its conclusions are a "logical consequence" of its premises. This answer is interesting, since it surfaces in it that term "logical" that supports our choice of defining logic as the study of the correct reasoning; still it is not very informative, for it is by no means obvious what "logical consequence" means. If we want to avoid a trivial circularity, this notion must be independent of the elaboration of a logic understood as a discipline and, moreover, should be the touchstone for evaluating the soundness of this discipline. The most spontaneous answer to our question seems to be that the logical consequences of certain premises are those propositions that follow from them according to the laws of thought, and this has actually been for centuries the tacit conviction underlying the construction of logic, a conviction that suggested the impression that there is essentially only one logic (that can include several chapters), more or less in the same sense as there is only one geometry, articulated into different chapters. This idea could be pushed so far as to affirm that logic had no history and was completely created by Aristotle, since the subsequent developments could be considered simple details having to do more with the elegance of the presentation than with real novelties. ${ }^{3}$ The situation changed radically when the formalistic spirit that was gradually penetrating mathematics in the second half of the nineteenth century affected also logic, with the creation of the first logical calculi.

\footnotetext{
${ }^{3}$ This curious position was expressed by Kant, who was not very learned in historical matters, but was then taken as a working prejudice in the monumental work of Prantl (1855-1870), who collected and studied an enormous documentary material with the aim of proving that Western logic had been essentially an elaboration and often a regression with respect to the Aristotelian logic.
} 


\section{THE PLURALITY OF LOGICS}

The question of the plurality of logics has been lively discussed, especially in the first decades of the twentieth century, mainly as a consequence of the construction of several logical calculi. These were the expression of the sharp formalistic outlook that inspired mathematical logic and brought to considering logic itself as a purely syntactical discipline. The famous statement of Rudolf Carnap: "in logic there is no moral"4 (that is considered as the motto of logical conventionalism) wanted to stress that the same mature "modern" awareness - that had obliged people to overcome, for example, the old view that there is just one "genuine" geometry (that is, the geometry), after the construction and the full acceptance of the non-Euclidean or non-Archimedean geometries - had to be applied to logic too. Therefore, there is no "genuine" logic (or the logic), but many possible logics, whose admission is suggested (but not imposed) by practical considerations. The defenders and the opponents of this view were often involved in a rather sterile dispute, for lack of sufficient analysis, and for an excess of polemic spirit.

The polemic attitude was produced by the claim of certain defenders of the new logic (or logics), according to whom this had "falsified" or overcome the traditional logic (for example, Withehead and Russell maintained, in the Principia Mathematica, that the Aristotelian syllogistic revealed several mistakes, if submitted to the exact formal tools of mathematical logic). Or, in the case of more tolerant positions, it was maintained that the new logical systems were "at variance" with the old logic. The opponents of the pluralistic view sometimes tried to show that the traditional logic was by no means wrong (for exam-

\footnotetext{
${ }^{4}$ Cf. Carnap (1934) where the famous "tolerance principle" is posed from the beginning as a basic view in the interpretation of the spirit of logic. The quoted statement occurs in $\S 17$ of this work, specifically devoted to "The tolerance principle in syntax".
} 
ple, Lukasiewicz was able to clarify why the Aristotelian syllogistic was correct from the point of view of mathematical logic, provided certain philosophical presuppositions of this syllogistic be duly recognized (Cf. Lukasiewicz (19572)). Sometimes they tried to "recapture" the features of the new logics within the framework of the traditional one, or maintained that certain recalcitrant features of the new logics should be ascribed to the "methodology" of certain particular sciences, and not to logic in a proper sense.

The lack of sufficient analysis we have mentioned consists in the fact that no preliminary clarification was carefully proposed as to the very meaning of logic. If such a clarification had been provided, the debated issue could have been seen not as an "aut-aut" question (one logic, or many logics), but as a question admitting a double answer: there is a sense according to which logic is unique, and another sense according to which several logics are legitimate. In this paper we shall try to outline such a diversified answer.

To give a rough idea of the solution we are going to propose, we could say that we want to present a view of logic comparable with the view of geometry maintained in the celebrated "Erlangen Programme" of Felix Klein (1872). The best known part of this programme consists in the group-theoretic treatment of the different geometries (elementary, metric, projective, affine, etc.) that permits to uncover a strict and almost genetic relationship among these theories, which puts them on an equal footing as to their mathematical legitimacy. Nevertheless this does not imply that whatever consistent mathematical theory is a "geometry", in spite of its being expressible in group-theoretic terms. Klein explicitly distinguishes between "geometry" and simple "mathematics", and maintains that only those theories are geometries, whose group "preserves" the "fundamental group" (that is, the group characterizing elementary geometry). In this way it is possible to maintain the unicity (or specificity) of geometry, with reference to its most stan- 
dard part, and at the same time to justify the plurality of geometries. A serious exploration of this Kleinian analogy, however, would be technically too complicated, and lead us to far afield. Therefore, we shall be content with this general hint.

\section{THE NOTION OF LOGICAL CONSEQUENCE}

If we try to explain what it means that a proposition $B$ is a logical consequence of another proposition $A$ (to consider the simplest case), we could say that one feels obliged to admit $B$, once one has admitted $A$. This kind of explanation considers the link of logical consequence as a particular form of psychic constraint, like an irresistible mental inclination (and this seems well in keeping with the idea that logic studies the movements or articulations of thinking). Nevertheless anyone certainly remembers some occasions on which he or she had believed a certain $B$ to be a logical consequence of a certain $A$, and then had recognized that $B$ was not really a "logical consequence" of $A$. This indicates that the link of logical consequence has a psychic "counterpart", but does not precisely consist in this; that it is something much more "objective", whose nature should be looked for in "thought", rather than in "thinking". In order to capture this objective feature one could say that $B$ is a logical consequence of $A$ if "it is not possible" to admit $A$ and reject $B$. This answer, however, is far from clear, since it is rather mysterious what this impossibility actually amounts to.

A very convenient way out of this impasse consists in considering the primary nature of propositions (whose linguistic expression are the "declarative sentences"): a proposition is the content of a judgment (that is, of a particular form of thought) whose primary characteristic is that of being either true or false. It is not essential for the sake of our investigation to find an agreement about a "definition" of truth: we can safely admit the definition that is implicitly presupposed by whatever 
"theory of truth" (since all such theories rather propose different "criteria" for truth $)^{5}$, and that consists in saying that a proposition is true if and only if that aspect or item of reality to which thought intends to refer is actually such as the proposition says. Now there are cases in which this aspect or item of reality is immediately present to our thought, but humans have spontaneously and unconsciously uncovered that, thanks to their thinking, they are endowed with the marvellous capability of attaining truth even in those cases in which this is not immediately present, since they are able to capture this truth by means of their reasoning. This reasoning is able to establish certain links between propositions that are "truth-preserving", that is, that lead from true propositions to other propositions that are also true, in spite of not being immediately true.

The practice of intuitive reasoning, however, in particular when the conclusion is derived from a set including several premises, easily shows two things: sometimes the reasoning seems impeccable, but the conclusion is patently false; sometimes a "form" of reasoning that has led to true conclusions in certain cases, leads to a false conclusion in a different case. In the effort of understanding these facts, and once we can exclude that some premises admitted as true were in fact false, we may find out that the conditions under which the premises (or some of them) were recognized as true are not the same as those under which the conclusion can be true. This leads to a refinement of the notion of logical consequence, and we can stipulate that a proposition $C$ is a logical consequence of a given set of premises $P=\left\{P_{1} \ldots P_{n}\right\}$ if and only if in all the cases in which all the premises are true, also the consequence $C$ is true exactly under the same conditions.

\footnotetext{
${ }^{5}$ Here, again, one should note that the distinction between definition and criteria for truth has been the subject of a large literature, in which very different positions have been advocated, but it is not important for the sake of this paper to enter this discussion.
} 
This definition may be satisfactory, but is unmanageable, it offers no "criterion" for ascertaining if and when $C$ is a logical consequence of the set $P$, since it would imply something like an infinite control of all possible truth conditions of $P$. A solution of this difficulty seems available: if $C$ has been reached by means of a reasoning, and reasoning has been understood as a "truth-preserving" linking among propositions, the truth of $C$ would be granted if $P$ is true. This solution is viable, however, only if, among the many sorts of reasoning humans spontaneously adopt, we are able to single out the correct reasonings, that is, those links that necessarily lead from true propositions to true propositions. Therefore, if we find that a certain "form" of reasoning had permitted to attain true conclusions from true premises in several cases, but failed to do so in other cases, we must say that the true conclusions were not obtained in virtue of the reasoning, but only accidentally or contingently, since the reasoning was not correct (following Aristotle, we could say that it was simply a "sophistical" argument).

We have come, finally, to an acceptable characterization of logic: it is a theory of the correct reasoning and, for this reason, it cannot be understood simply as a descriptive enterprise. Sure, in order to concretely construct logic, we must take into consideration human reasoning or "thinking", but in this thinking we find quite commonly used incorrect reasonings, and in order to discard them we need a meta-reflection in which the necessarily truth-preserving forms could be singled out and explicitly codified. This is the normative aspect of logic, that entitles its being also called the investigation of the "laws of thought". The use of "thought" instead of "thinking" underlines its objective nature, while the term "laws" hints at its features of universality and necessity, that correspond to the level of idealization that is inherent to logic as well as to any intellectual construction (as we will better see in the sequel).

Manuscrito - Rev. Int. Fil., Campinas, v. 34, n. 1, p. 51-88, jan.-jun. 2011. 


\section{THE BASIC STATUS OF ALETHIC ${ }^{6}$ LOGIC}

The analysis outlined thus far has shown logic coming out of the reflections on truth, and on the notion of logical consequence conceived as strictly related to the investigation of truth. This is tantamount to saying that something like an "elementary logic" has emerged as the basic framework of logic as such. This framework has the features of a programme that must be patiently and carefully developed, and for this reason it is necessary to pass from thought to its explicit linguistic expressions. We must proceed to several idealizations, for example by first summarizing under a few "sentential connectives" or operators the many immediate links among sentences we use in everyday language, or by singling out certain "quantifiers", and so on. In brief, we must outline a "logical grammar" of the (idealized) language. We then proceed to explore more complex links among propositions, that correspond to arguments or reasonings in a proper sense, and we begin finding out certain general patterns of them. The most elementary are those in which the "truth-preserving" characteristic of the links results from considering sentences simply as linguistic entities that are either true or false, and the explicitation of such patterns constitutes the domain of sentential logic. It is easily found, however, that we use a lot of correct arguments whose patterns cannot be captured by sentential logic: not even a very elementary syllogism can be recognized as correct on the ground of this logic, since it presupposes a consideration of the "terms" entering a sentence, and the correct logical link among the sentences depends on certain correct links among the terms.

\footnotetext{
${ }^{6}$ We explicitly stress that we shall use the term alethic in a very general sense, immediately related to its etymology (aletheia $=$ truth $)$, in accordance with our proposal of strictly linking logic with the investigation of truth conditions. In the literature this adjective has a much more restricted meaning, since it is used to indicate the basic modal logic, and distinguish it from other "structurally similar" modalities, such as, for example, "deontic" modalities (cf. von Wright (1951)).
}

Manuscrito - Rev. Int. Fil., Campinas, v. 34, n. 1, p. 51-88, jan.-jun. 2011. 
We can call syllogistic (or "categoric syllogistic") such a deeperlevel logic, and try to determine all the patterns of it that corespond to correct reasonings: something Aristotle had done. believing to have captured in this way the ideal forms to which every correct reasoning is reducible. Already medieval logicians, however, had noticed that even such an elementary and obviously correct reasoning as the following: "the circle is a figure, hence who draws a circle draws a figure" is not fully compressible in any of the standard syllogistic "figures", in spite of having a shape vaguely resembling that of a syllogism. We know that the reason of this fact is that traditional syllogistic only considers properties, but not relations (in modern terminology we would say that it corresponds to a logic of classes or of monadic predicates), and this also explains why it was never concretely used in the exposition of mathematics, where the consideration of relations is no less essential than the consideration of properties in the proof of even the simplest theorems.

The situation just mentioned is quite interesting: during a very long historical time it was believed that logic had been completely developed (this was for example the opinion of Kant), though it only contained syllogistic with a few complements of sentential logic and modal logic (that were mainly related to the treatment of hypothetical and modal syllogisms). A great deal of correct reasonings adopted in everyday discourse and several sciences (including mathematics), however, followed patterns that essentially overstepped those of syllogistic, but people seemed unaware of this, being convinced that any correct reasoning could "in principle" be reshaped as a sequence of syllogisms, though this reelaboration would be too cumbersome in practice to deserve being performed. 


\section{MATHEMATICAL LOGIC AND ITS DOUBLE MEANING}

Things changed deeply with the construction of mathematical logic in the 19th century. Two meanings have been attached to this denomination. According to the first (that we can refer to Boole for simplicity), it indicates the fact that logic has been reformulated in a "mathematical way" (that is, essentially, in the form of symbolic calculi). According to the second (that we can refer to Frege in a similar way), it denotes the "logic of mathematics". From the point of view of the "external appearence", the first meaning expresses the most significant "novelty" with respect to traditional logic. Yet it is not so: exposing logic in the form of symbolic calculi was simply the concrete application of a point of view already present in the Posterior Analytics, and explicitly advocated by Leibniz. It was the programme of submitting the common reasonings of mathematics to a rigorous logical study, instead, that led to uncover the complexity of such reasonings, and eventually to develop new logics (such as the logic of relations, the logic of classes, the predicate logic, and so on), that included authentic "novelties" with respect to traditional logic (novelties that could be suitably expressed in certain calculi, as Frege and other logicians were able to do).

This story lends itself to a few interesting reflections. In the first place, one can see here an historical example that "science may need new logics", for it was in the effort of making explicit the correct reasonings applied in a particular science (that is, mathematics) that genuine new logics (new with respect to traditional logic) were found. Yet one can legitimately say that this was simply a de facto circumstance, since such new logics are indeed needed not only for rigorously analyzing mathematical reasonings, but also a great deal of reasonings of common sense. A more subtle remark is the following: is it really correct to say that "new logics" were discovered, instead of saying that "new branches of logic" were developed? The second alternative seems indeed more appropriate and, as a matter of fact, we usually consider, 
for example, sentential logic, first order predicate logic, second order predicate logic, and so on, as distinct but not separate chapters of one logic, corresponding to different "levels" of the logical analysis of language. Moreover, the "older" parts of logic are "included" in the new branches (for example, categorical syllogistic is contained in first order predicate logic, that "in addition" is also able to consider polyadic predicates, or relations, and the correct reasonings in which they occur). In other words, the different logics behave like the different geometries in the approach of the Erlangen Programme: they must all contain something like the "fundamental group" (that is, traditional logic), and are characterized by properties that from a certain point of view are broader, and from another point of view are more restricted than those of the "inferior" levels (precisely as it happens in the case of the group-theoretic hierarchy of geometries). For example, sentential logic corresponds to a linguistic level endowed with a low "expressive power", but is correct, complete, and decidable; first order logic is endowed with a greater expressive power (individuals, properties and relations are distinguished, quantification over individuals is admitted), but, while correctness and completeness are preserved, decidability is lost in general (that part which corresponds to traditional logic, that is, the logic of monadic predicates, however, is decidable); second order logic is even more expressive (properties of properties, properties of relations, relations between properties are considered, quantification over properties is admitted), correctness is preserved, but completeness and decidability are lost.

Without going into additional details we can conclude: in spite of the fact that, historically speaking, several new branches of logic were discovered and developed as a consequence of having concentrated the attention on the correct reasonings of mathematics, these new branches cannot be said to be intrinsically dependent on the needs of mathematics. They rather constitute the completion of lacking chapters of logic 
as such: we can call "logic" any single chapter (and characterize it by means of an adjective or a specification), this fact, however, does not really mean a plurality of logics, but rather an articulation in parts or subdomains of the unique logic that has been characterized (we must not forget it) as an effort for making explicit the links corresponding to the relation of logical consequence. Therefore, we can say in brief that the domain of logic has appeared to coincide, up to now, with that of alethic logic.

\section{THE VARIETY OF LOGICAL CALCULI}

The thesis of the plurality of logics, as we have already seen, was especially advocated, in the first decades of the twentieth century, as a consequence of the existence of a great deal of logical calculi, and of the unlimited possibility of constructing new ones. Owing to the strict syntactic and formalistic point of view prevailing at that time, any such calculus was considered to be a logic in itself, and the plurality of logics was indeed consistent with this view. Yet a rather simple reflection shows the inadequacy of such a perspective. It is enough to consider that a calculus (as it has been clear since Leibniz, and repeatedly stressed afterwards) is nothing but a system of rules for operating with symbols. This does not entail, however, that whatever calculus is a logical calculus (for example, chess and bridge are games whose very explicit rules justify considering them as calculi, but they are never listed among the logical calculi). Already Leibniz had said that, among the many varieties of calculi, it was possible to single out a particular one, that he called calculus ratiocinator, whose specific nature was that of reproducing the features of correct reasoning. At first sight one would be inclined to say: very well, Leibniz was convinced that logic is mirrored in a single special calculus, we accept his point of view and say that, if we nowadays admit several logical calculi, we 
are obliged to admit that each of them correspond to a definite logic, and therefore we admit several logics.

Things, however, are not so simple. The fact is that, once we have "freely" constructed a calculus, we must prove that it satisfies certain conditions, before recognizing it as a "logical" calculus, and these conditions are the same for all calculi. They consist essentially in a "faithfullness" with respect to the notion of "logical consequence", in the following sense. First of all we must be able to show that the symbols and rules of the calculus can be "interpreted" in such a way that (globally taken) they can be seen as expressing sentences, and links among sentences. Then a metatheorem must be proved: it must be shown that, by using this calculus, only logical consequences can be derived from any set of premises. This minimal requirement amounts to recognizing that the calculus is correct, and a calculus not satisfying this requirement is excluded from the outset from the domain of logical calculi, since it would permit to derive from a set of premises certain conclusions that are not a "logical consequence" of the premises. Besides this indispensable condition, another appears as a highly desirable requirement for a calculus: that this permits to derive from any set of premises the set of all its logical consequences. This requirement is indicated as the semantic completeness of a calculus. We know that only for calculi corresponding to sentential logic and first order logic completeness can be proved, while higher order calculi fail to satisfy this requirement. The fact that these two metatheorems are taken as a criterion for admitting a calculus as a "logical" one, and that this criterion applies identically to all the calculi "corresponding" to a given logic, already indicates that the plurality of calculi does not mean a plurality of logics. ${ }^{7}$ The analogy with geometry is again instructive for

\footnotetext{
${ }^{7}$ We can note, incidentally, that certain authors (among them, Quine and Kneale) were so severe in requiring the full respect of the two said conditions as to exclude from the realm of logic second-order logic.
} 
understanding this point. Elementary geometry and projective geometry are certainly two distinct geometries: each one of them, however, can be axiomatized in many different ways, and we know several axiomatizations of the elementary geometry, or of the projective geometry (mathematicians of the end of the 19th century, and in particular Peano and his school, have shown a great skill in producing such axiomatizations). We can express this fact by saying that, for example, elementary geometry, though being one, can be presented or formulated under many different "forms", and we can explain the meaning of this assertion by saying that all these forms are "equivalent", in the sense that precisely the same geometrical truths can be proved as theorems in each of these axiomatic systems. In the very same way we can say, for example, that all logical calculi corresponding to a given "logic" (sentential, first order, etc.) that are correct and complete are "equivalent" in the sense that they permit, from any set of premises, to derive exactly the same conclusions. This fact is indeed obvious: let a set of premises $P$ be given, and a sentence $S$ that is derivable from $P$ using a calculus $K$ and not derivable using another calculus $K^{\prime}$. If $S$ is a logical consequence of $P$, then $K^{\prime}$ would not be complete; if $S$ is not a logical consequence of $P$, then $K$ would not be correct.

The general conclusion we have reached up to now can be summarized as follows: in a proper sense one should say that there is one logic (alethic logic), articulated into several domains, each domain being in turn expressible or "formalizable" by means of different logical calculi. Using a systems-theoretic approach this view can be axpressed by saying that logic is a global system whose subsystems are the particular logics, while the different calculi do not constitute further subsystems, but only different descriptions of the functioning of a given subsystem. According to a different approach we could say that there is a general concept of logic (corresponding to the notion of alethic logic), that is "exemplified" by several particular alethic logics (sentential, first order, 
second order, class logic, etc.), that are formalizable by means of different logical calculi.

In both ways we can see how it is possible to maintain the uniqueness of logic (from a certain point of view) and the plurality of logics (from another point of view) in a consistent way. What we have seen, however, still remains at the margins of the real debate concerning the plurality of logics: we have only refuted the thesis that such a plurality is constituted by the mere existence of a plurality of logical calculi. The core of the debate, however, consists in accepting or rejecting the thesis that alethic logic is the only or the genuine logic. We shall now proceed to explore this issue.

\section{THE BROADENING OF THE DOMAIN OF LOGIC}

The path we have followed for determining the nature of logic has been that of analyzing the notion and the patterns of correct reasoning, as it is intuitively understood, and the most basic requirement we have found is that any correct reasoning must be truth-preserving. Indeed we have defined the notion of "logical consequence" with explicit reference to truth, then characterized a correct reasoning as that in which certain propositions are linked with their logical consequences, and finally have characterized logic as the idealization and explicitation of the links realized in correct reasoning. In this effort of idealization and explicitation the focus on the "links" (as we have already noted) led to disregard the meaning, and even the contingent truth, of the sentences actually occurring in a discourse, especially because these are often the source of fallacies in everyday reasoning. Yet human reasoning, being a part of the activity of thinking, never takes place in a vacuum, but is always unfolding within a context of meaning.

This, in particular, is reflected also in the intuitive notion of logical consequence, for which we usually require not only that premises and 
consequences are equally true, but also that the premises are "relevant" to the truth of the consequences. This fact was taken into account by traditional syllogistic, whose basic principle was: quae conveniunt uni tertio conveniunt inter se (things that are pertinent to a third thing are mutually pertinent). The two premises of a syllogism must contain a common "medium" term, and at the same time be true: a double condition that grants that they are really intrinsically related, and for that reason really relevant to the conclusion (that consists in a sentence containing only the two "extreme" terms). Indeed the venatio medii (the "hunting" of the appropriate medium) was considered as the most challenging part of the construction of good arguments. In contemporary logic, the discussions on material and strict implications, and especially the construction of relevant logics constitute examples of a "broadening" of the horizon of purely alethic logic, that has led to logical systems that are not certainly "at variance" with alethic logic, but introduce stricter requirements for the "logical" inferences. A good survey of the construction of relevant logics can be found in Belnap (1981).

That the consideration of the meaning is by no means just accessory or contingent in the formation of correct reasonings is clearly shown by the fact that several "immediate inferences" in our reasoning are simply the explicitation of certain meanings, from which more complex inferences can be costructed. For example, we consider an immediate "logical consequence" of affirming that a certain event was "necessary", to affirm that it was "possible" (but not vice versa); we consider a "logical consequence" of affirming that a given action is "obligatory" to affirm that its omission is "forbidden"; we consider a "logical consequence" of affirming that one "believes that $p$ " that he does not "believe that not- $p$ ". If we consider these immediate inferences (and many other of a similar kind), we recognize in them the basic feature of the alethic notion of logical consequence (that is, it cannot happen that the premise 
is true and the consequence is false), but we cannot say that the consequence is true in virtue of any linguistic link, just because there is no such link here (these are all examples of immediate inferences). The actual situation is that such inferences are, so to speak, simply the linguistic explicitation of a particular "conceptual domain", in which certain basic notions "interdefine" each other in a way quite similar to that in which truth and falsity are mutually interdefined.

The three examples just presented obviously hint at immediate inferences belonging, respectively, to modal logic, deontic logic, epistemic logic, and have each a definite specificity, the specificity of a given "conceptual domain" that is possible (and useful) to render explicit, for example, through an appropriate axiomatization. The above examples could in fact be single items of such axiomatizations, whose function is comparable to that, let us say, of the axioms of the familiar alethic sentential logic. The specific nature of every conceptual domain, however, entails that differences be no less significant than similarities. For example: it is fully in keeping with the notion of truth that it has just one opposite, that is, falsity. Therefore alethic logic must consider only two "statuses" of a proposition (true and false), and the "bivalence principle" is appropriate to it. But if we consider the conceptual field of modality, it would be arbitrary to impose to it something like a bivalence principle, since to a state of affairs can be attributed not two, but three modalities (possible, impossible, necessary). Similarly, from a deontic point of view, an action may be not only obligatory or prohibited, but also permitted. These considerations indicate that a modal logic and a deontic logic should be "three-valued" rather than "two-valued", with the awareness, however, that these "values" must not be considered as "truth-values" (a confusion that has affected several controversies). ${ }^{8}$

\footnotetext{
${ }^{8}$ This feature is obviously compatible with the fact that, from a formal point of view, it is possible to take only one of such notions as primitive, and
} 
The explicitation of these logics is delicate because they keep a continuous interplay with alethic logic: in the first place, because the axioms of these logics are meant to be an explicitation of what modalities, deontic aspects, epistemic attitudes, truly are. In the second place because it is possible sometimes to translate in terms of true propositions the contents of axioms that do not refer to propositions (for example, instead of saying: "the state of affairs $A$ is necessary", we can say: "the proposition $S$ is necessarily true", where $S$ is the linguistic description of $A$ ). Finally, axioms of a given logic may concern propositions, and yet not reflect all the features of a propositional logic. For example, we have given above the "epistemic" statement: "if $A$ believes the sentence $S, A$ does not believe the sentence not- $S$ ". The soundness of this statement relies: upon the epistemic fact that believing a proposition is equivalent to believing that it is true; upon the alethic fact that the negation of a true proposition is false; upon the alethic fact that one cannot believe at the same time that a proposition is true and false. All this, however, does not entail the validity of a principle of bivalence in epistemic logic. Indeed, while it is correct to say that "one never believes $\mathrm{S}$ and not-S", it is not correct to say that "one always believes either $S$ or not- $S$ " (since one may well "suspend" belief in the case of some particular $S) .^{9}$

In the above examples our reasonings regard, so to speak, clear-cut cognitive situations, but in many cases we are obliged to formulate correct reasonings in less privileged cognitive situations, and we are indeed able to do so. For example, the clear-cut partition of modal logic according to which an event is either necessary, or possible, or impossible, is rather unsatisfactory for several purposes, in which we are interested

define the others by a suitable use of negations. This fact does not eliminate the other fact, that these notions are conceptually distinct.

${ }^{9}$ Other well known examples are offered, for instance, by deontic logic, where it is clear that, in spite of its "structural analogy" with modal logic, this analogy is not total (cf. von Wrigt (1981)).

Manuscrito - Rev. Int. Fil., Campinas, v. 34, n. 1, p. 51-88, jan.-jun. 2011. 
in evaluating the "measure" of the possibility of an event: probability calculus is, from this point of view, a "logical" response to this legitimate desire, in the sense that it permits to give such a response not just on the basis of a guess, but of rigorous and elaborated reasonings. ${ }^{10}$

Already in traditional epistemology the term "probable" was used not for denoting some modal property concerning the occurence of an event, but for indicating a particular state of mind with regard to the truth of a proposition: in that context it was usual to indicate as "ignorance" the lowest degree, with "doubt" the situation in which the mind has not taken a position yet, with "opinion" the state in which the mind is inclined to admit the truth of a certain judgment, with "certainty" the state in which the mind is in full possession of the truth. Opinions, being always affected by a certain degree of uncertainty, were said to be more or less "probable". Therefore probability was meant to admit of an indefinite spectrum of degrees, spanning from ignorance to certainty. ${ }^{11}$ It is rather natural to translate this qualitative traditional view in terms of the modern notion of probability, by identifying ignorance with a probability $p=0$, certainty with $p=1$, and all the intermediate states with probabilities $p_{i}$ with values comprised between 0 and 1 . Through such a translation it is also sensible to use the probability calculus to express the "logic" of such mental attitudes towards truth.

It would be mistaken, however, to consider such probabilistic logics as "at variance" with alethic logic, or as admitting a continuous spectrum of truth-values. For example, if we say: "it is $90 \%$ probable

\footnotetext{
${ }^{10}$ In the title of the already cited Laws of thought, Boole already puts the calculus of probabilities on the same footing as logic.

${ }^{11}$ This classical doctrine is standard in all textbooks of the "scholastic" tradition, including quite recent ones. For example, it is presented in Verneaux (1959), pp. 93-93. In the 18th century it gave rise to several developments regarding the "probability of judgments", where the tools of the recently born probability calculus in its "Pascalian" version were applied.
} 
that Peter is at home in this moment", we cannot avoid that the event mentioned in the proposition (that is, the presence of Peter at home now) either occurs or does not occur (bivalence principle in ontological sense), and from that it follows that the proposition describing the event is either true or false (bivalence principle for declarative logic); the probability of $90 \%$ only indicates that we very strongly adhere to the truth of the said proposition (though not feeling completely certain about it).

The last considerations offer us a guideline for understanding the "logic" of the unfolding of different logics. If we go back to our original considerations, in which we have seen logic coming out of the needs of the investigation of truth, that is as a powerful instrument (this is the meaning of the Aristotelian "organon") in the acquisition of knowledge, we can easily understand how the same Aristotle indicated the ideal conditions in which the use of such an instrument can give the best results: these conditions are those in which the reasoning starts from premises that are "immediately true, better known than the conclusion and cause of it" (Post.Anal., A, 2, 6). This is tantamount to saying that the ideal situation is that in which the reasoning can develop "under conditions of certainty". Humans, however, are obliged most of the time to develop their reasonings "under conditions of uncertainty", and the same Aristotle, in presenting the general aim of his syllogistic, precisely declared that his work "has as purpose that of finding a method for constructing syllogisms on whatever proposed problem, moving from probable premises" (Topics A 1, 100a 22). This means that, even if we have at our disposal an instrument (such as alethic logic) that is "sure" and yields truth if applied to true premises, we still remain with the task of evaluating to what extent the "probable" premises of which Aristotle speaks are actually probable. 
The methods and reasonings by means of which we try to establish the degrees of confidence in a given sentence (that is, in the truth of a given sentence) can legitimately be called a "logic", since they must necessarily move from certain true propositions, and lead us to other propositions we intend to be true, but whose truth is not absolutely granted (like in the case of standard alethic logic), such that we want at least to know "how confident" we can be that they are true. Inductive logic is the most important example in this domain, and certain criticisms that have been levelled against it are essentially misplaced: its aim is often pictured as that of coming to general conclusions from a finite (though perhaps very large) set of singular true premises, and it is too easy to note that such a transition is never absolutely granted. This is, however, a misrepresentation of the aim of inductive logic. Even in the case of enumerative induction, there is no pretention of attaining an absolutely true general conclusion, but only one of which we try to establish the "probability" in the traditional sense. That in this effort the probability calculus can be of great help is simply obvious, though certain precisions must be elaborated regarding the different meanings of probability that apply in this enterprise, and their relation with the notion of the "probability of a sentence". For this variety of meanings of probability cf. Agazzi (1988b). One must be aware, however, that inductive logic does not reduce to this application of the probability calculus, nor does it have as main purpose that of providing some justification for generalizations. In fact, inductive methods are used in several sciences (and also in non scientific contexts) to investigate some possible causal relations among events, on the basis of an available empirical evidence. In these cases we can speak of the "inductive support" provided by such evidence to a certain hypothesis, and it is possible to elaborate and formalize a "logic" of such a support that is not isomorphic with probability calculus. ${ }^{12}$

${ }^{12} \mathrm{~A}$ very instructive presentation of these different aspects is offered in Manuscrito - Rev. Int. Fil., Campinas, v. 34, n. 1, p. 51-88, jan.-jun. 2011. 
As a last example of a logic corresponding to the needs of reasoning under conditions of uncertainty we shall mention the circumstance that even the possibility that the premises from which we start are to some extent mutually inconsistent does not imply the collapse of our correct thinking. Paraconsistent logics have actually shown how correct reasonings can be developed within contexts in which a certain degree of inconsistency is present (apart of more technical applications, this logic is rather close to the familiar situations of our dayly reasonings, in which we are often far from sure that the system of our premises is really consistent).

\section{LOGIC AND THE OBJECT OF DISCOURSE}

Our considerations are a development of the remark stated above that reasonings cannot occur "in a vacuum". This is the obvious conse-

Cohen (1981), where it is shown that the application of the "Pascalian" approach to the evaluation of the soundness of a scientific proposition (that is, essentially, the use of standard probability calculus for improving the acceptance of this proposition), that characterizes Carnap's inductive logic, is opposed to Poppers doctrine according to which a hypothesis is the more valuable the more improbable it is. In spite of being opposite, these doctrines are shown to be appropriate, respectively, for expressing the degree of appreciation that is given to a scientific proposition, in the first case, from the point of view of a "tehcnological" purpose or, in the second case, from the point of view of a theoretical "explanatory" purpose. By deepening the analysis of the different systems of inductive logic elaborated in the past decades, Cohen shows certain shortcomings that can be eliminated in a "logic of inductive support" (that he has presented especially in Cohen 1970, 1977) which is not formalizable according to a "Pascalian" approach, but is formalizable using a generalization of a certain system of modal logic (the S4 system of C.I. Lewis). This discussion exemplifies very well how certain logical systems have been created in order to make explicit and controllable some rational arguments applied in the context of scientific research (and even according to different goals pursued in this research), and how they had to challenge several problems of a genuine formal logical nature. 
quence that reasoning is a particular activity of thinking, and thinking cannot be "thinking of nothing"; in fact the activity of "thinking" is inseparable from the production of "thoughts" that are its very actualization and have that "mental reality" which we can equate with their sense. When we say that thinking cannot be thinking of nothing, however, we do not mean that thinking is thinking of thoughts or senses (since thoughts are not the "content" of thinking, but simply thinking in act). Hence we must say that thought cannot be thought of nothing, and by this we underline that intentional nature of thought that has been analized several times by past and contemporary philosophical schools. It is at this stage that we can (and must) speak of an "object" of thought, and this is that to what thought refers (or its referent).

These clarifications are not just a pedantic digression, but a useful reminder for our discussion, since truth comes about precisely in this referential situation: it is the property of a thought whose content (sense) conforms to its intended object or referent. We are not obliged to find an agreement in specifying in what this "conformity" consists: it is enough to note that, in the same way that a thought necessarily is thought of something, also a proposition is necessarily true (or false) of something (that is, of its referent). In other words, a proposition is never true or false "in itself", but always relatively to a certain referent. What we have been saying about thoughts and propositions can be transposed to their linguistic expressions, that is, discourse and sentences. The conclusion is that an investigation of the truth conditions of a discourse cannot avoid taking into serious consideration also the particular nature of the objects of that discourse, since it is "in virtue" of such objects that a suitably constructed discourse can be true, and never "in virtue" of itself. ${ }^{13}$

\footnotetext{
${ }^{13}$ This problematic has been deeply analyzed in the large literature on "truth-makers" that we cannot discuss in this paper.
} 
The last affirmation seems contradicted by the existence of propositions that are usually considered as "true in themselves", or "always true": typically the propositions of logic, also called "logical laws". Yet even in such cases these propositions can be said to be true because, through a reflective act of thinking, we can take their meaning as the object of reflection, and recognize that "in virtue" of this meaning, they represent a universal scheme or "form" that will yield true propositions not "independently of any interpretation" on particular referents, but "in connection with whatever interpretation" on particular referents. For example, the sentence: "if all $A$ are $B$ and all $B$ are $C$, then all $A$ are $C$ " can be said to be true in itself, or always true, only if a suitable meaning is attached to all its linguistic ingredients: sentential connectives must receive a certain meaning, not less than the quantifier "all", while $A, B$ and $C$ must receive the meaning either of names for classes or of names for properties; finally the meaning of "are" must be clarified: it can be taken as expressing essentially a class inclusion, or a predication and, in both cases, the "universal truth" of this logical law will be established "in virtue" of the intuitive meaning of such fundamental intellectual operations as the assignment of individuals to a class, or of the attribution of a property to individuals. In conclusion, the said sentence is true of a (rather complex) network of thinking operations whose description contains some "empty boxes" (the letters $A$, $B, C)$ : if these boxes are filled with contents not of any kind, but of the kind prescribed by their meaning, the result will necessarily be a special true proposition about those particular contents.

\section{MEANING, SENSE AND REFERENCE}

Our considerations might sound rather peculiar to many readers, since we are maintaining that meanings can become the object of a certain kind of knowledge, and this seems to ignore the distinction be- 
tween sense and reference that we have accepted in a preceding part of this paper. This is, however, just a superficial first impression. In fact, "sense" is normally understood as a mental representation, while "referents" are understood as concrete material "things" to which a certain sense can be properly related. This distinction (that in modern times was elaborated especially by Frege) is opportune, but it cannot be taken as a separation, and in order to stress the strict relation existing between sense and reference we explicitly speak of meaning as a composite unity of which sense and reference are the constitutive parts. This can be seen if we consider that any sense unavoidably "points to" or "intentionally refers to" an entity to which it applies, though this entity may happen not to exist concretely. This situation can be expressed by saying that a sense encodes (through the presence of a set of concepts) a specific class of entities, and these entities exemplify the sense.

In several particular cases sense can be constructed "artificially", and we proceed to investigate whether the class of referents it points to is empty or not. For example, in mathematics we can give a "definition" (explicit or axiomatic) by a coordination of certain concepts, and then look for possible "mathematical entities" that satisfy this definition. But even in comon language we can construct expressions such as "golden mountain", or "the present king of France". It would be improper to say that they are "meaningless", because they can be understood, and hence have a sense, and it is in virtue of this sense that we can find out that they have no reference. This happens because this sense entails that possible referents should be endowed with certain properties defining a given class of objects (in the case of the second example, these properties are in part implicit, in part explicit: for example, the presence of "the" indicates that the class must contain just one individual, it is implicit that the referent be a concretely existing human person, and not just a character in a novel, the adjective 
"present" indicates a temporal coordinate, while "king" summarizes a set of political and legal prerogatives). If it happens that no individual exemplifies all this joint properties, we conclude that the expression denotes an empty class, and that no referent corresponds to this sense.

In the majority of the cases, however, the sense is not created artificially, but is obtained (by means of the intellectual operation that we call "abstraction") from the "intentional presence" of certain referents that we encounter in some kind of experience. Moreover, the sense is not established once and for all, but is continuously "controlled" (in its explicit formulation), through a comparison with the "intended referents". This is why sense is just a part of the meaning, and it cannot be completely disjoint from the referents that exemplify it.

An important remark is now fundamental for our discourse: humans are endowed with the capability of directing their intentional power not only to material things, but also to abstract objects, and in particular to meanings and senses (this peculiarity was already pointed out in the distinction of intentio prima and intentio secunda proposed by medieval philosophers). In this intellectual activity sense and meaning become referents of the intentional act, they are the objects of a second-level investigation, and it is possible to acquire genuine knowledge about them.

In this way we have given some necessary clarifications for supporting what we have said above, when we have maintained that the different "logics" must be understood as explorations and explicitations of the reasonings we perform within particular "conceptual domains" that correspond to certain fields of meaning. But now this clarification can be further developed. We have just maintained that the unity of sense and reference (that is, the delimitation of a certain field of meaning) occurs in the context of a particular kind of experience. Unfortunately the concept of experience is usually affected by a too narrow empiricist characterization, according to which it means 
exclusively sense-experience. This narrow sense has been historically suggested by modern natural science and sanctioned by the authority of Kant. In common language, however, we normally use expressions such as "moral experience", "aesthetic experience", "religious experience", and so on. Are these expressions misleading? By no means: they indicate the basic fact that in our "contact" with reality we feel directly implicated, and induced to express judgments of specifically different kinds. For example, in front af a certain action we inevitably express the judgment that it is "good" or "evil" (and this judgment has nothing to do with an exact empirical "description" of this action); or we can judge a picture as "beautiful" or "ugly" (and, again, this does not correspond to any precise empirical description of the picture). Exactly as we "experience" a cherry "as" red (in a visual sense experience), we "experience" the action of helping a person in necessity "as" good in a moral experience, or a Schubert melody "as" beautiful in an aesthetic experience. (By the way, even Kant, who could not use the term "experience" in the context of morality, having restricted its meaning to sense experience in the Critique of Pure Reason, speaks of the presence of the moral law in every human as a "Faktum der Vernunft", as a "fact" of Reason, in the Critique of Practical Reason: a very vague and imprecise expression that actually hints at that feature of "givenness" that, even according to him, characterizes the immediateness and irreducibility of any experience).

We need not give additional examples and details. The above explanations may be sufficient for justifying the existence of different logics, and the justification is rather simple: whenever in a certain field of experience we are led to use reasonings or arguments for supporting judgments, the space for the explicitation of a specific "logic" is in principle open. It is a contingent fact that only a few of such logics have been actually constructed or sketched: this mainly depends on the "interest" we could have in rigorizing such reasonings, and this is only 
a historical fact. In certain fields, for example, we are still convinced that judgments are subjective, or can at most be supported by general and not stringent reasonings (like in the case of aesthetic judgments), and do not expect that a "logic" will be looked for. In other fields the interest for the explicitation of a logic has surfaced recently (like in the case of epistemic logic), and one cannot be a prophet in these matters.

There are domains, however, where the use of rigorous arguments is considered essential, and these are typically the sciences. Therefore it is sensible that some special logics have been proposed (or will be proposed) for particular sciences, or scientific theories. But in order to see this in depth we need to follow further our investigation.

\section{THE RELEVANCE OF THE REFERENTS FOR LOGIC, OR FROM PURE TO APPLIED LOGIC}

That logic cannot be reduced to a purely formalistic construction should be clear enough after all the considerations we have presented: being the explicitation of the forms of our correct reasonings, and reasonings being part of our thinking activity, logic cannot help being subjected to the variable "conditions" of thinking. Precisely this kind of consideration has led Kant to develop a "transcendental logic" (in which rather the "a priori conditions" of thinking were stated). Our intention here is not that of discussing the sense and the possibility of such a logic; when we mention the "conditions" of thinking we want simply to stress that our thinking activity inevitably depends on, or is immersed in, a variable cognitive context, and even if we maintain, for example, that the most fundamental part of logic is that which makes explicit the truth-preserving linkages among propositions, we might be in difficulty in applying this fundamental part of logic in certain cases, owing to the particular content of the propositions. 
This problematic situation was known already to traditional logic. For example, Aristotle discusses at length (in chap. 9 of De interpretatione) the possibility of assigning a truth-value to propositions such as "there will be tomorrow a sea-battle" (propositions referring to single future contingent events), and comes to the conclusion that such propositions are neither true nor false (the issue has been taken up several times by posterior logicians under the classical title "de futuris contingentibus"). This Aristotelian position is very interesting, since the same Aristotle had explicitly defined a proposition as a discourse that is either true or false ("not every discourse is a proposition, but only that discourse in which truth or falsity reside", De int., 4, 17a).

In spite of this he can consistently maintain that propositions regarding future contingent events are neither true nor false, because truth and falsity are properties pertaining to a judgment, in virtue of its way of expressing the "being" or "not being" of something, but it is not said that we can express such a judgment in all circumstances. There is, first, a cognitive aspect: future events cannot be directly known (they cannot be referents of an act of cognition); this does not totally exclude the possibility of saying something true about them, but only indirectly, when they can be the object of universal or necessary judgments. In the case of a future contingent event we cannot assign it (at present) neither existence nor non-existence, precisely because it is contingent, and contingency means the possibility of being and not being. This is an ontological consideration, that regards the particular nature of the referent of the discourse. From both points of view we come to the conclusion that a logic for the discourse about future contingents events must admit an intermediate value (or even a distribution of such values) between truth and falsity, that it might be equipped with some "temporal" parameters, and, briefly, be more or 
less different from usual logic: this owing to the cognitive and ontological peculiarity of the intended referents of the discourse. ${ }^{14}$

\section{THE LOGIC OF PARTICULAR SCIENCES}

This classical and relatively simple example offers a paradigm for a general situation: in every science the referents of the discourse are specific objects that are singled out and shaped by means of conceptual and operational tools, amounting at the same time to a characterization of their cognitive accessibility and ontological status. Therefore, it is altogether reasonable that the arguments we adopt in a given science be sensitive to such cognitive and ontological conditions, and can give rise, not in every case, but in certain cases, to special logics. A few well known examples can be mentioned as a confirmation of this.

Let us consider as first example elementary arithmetic, in whose modern rigorous presentations we find the principle of "mathematical induction". Even when this principle is formally expressed as a special "axiom", it must be recognized that it significantly differs from the other axioms, since it has a "logical" rather then a "numerical" nature in the following sense. While from the other axioms different properties of numbers can be deduced (for instance, the fact of being even, odd, prime, etc.), no numerical property can be deduced from this axiom, but it is used for proving that a certain property holds for all numbers in a distributive sense. Its logical nature was recognized, for example, by Poincaré, who interpreted it as summarizing an infi-

\footnotetext{
${ }^{14}$ The above considerations obviously indicate the motivation that has led to the construction of tense logics. One must be aware, however, that the simple purpose of taking time into account does not directly produce a unique type of tense logic. For example, the logic elaborated by Prior (using temporal operators), and that proposed by Quine (using temporal quantifiers) are different not only from a technical point of view, but also because they reflect very different epistemological and even metaphysical tenets (cf. Haak 1978, pp. 156-162).
}

Manuscrito - Rev. Int. Fil., Campinas, v. 34, n. 1, p. 51-88, jan.-jun. 2011. 
nite chain of syllogims. ${ }^{15}$ A confirmation of this fact is offered by the various generalizations of this principle. In set theory (where transfinite numbers are admitted and defined) we normally use "transfinite induction" on ordinal numbers. In mathematical logic several kinds of "proofs by induction" are used for proving a great deal of metatheorems concerning logical calculi (that can easily be presented as "generalized arithmetics"). It is not difficult to recognize that this principle is by no means "universal", but can be properly used only depending on the particular class of referents to which our reasonings are applied: only if this class is defined in a special way, that is, it is "constructively" defined, we can use a form of inductive proof (and also of "inductive definition") for treating the properties of its members. A class of entities is constructively obtained if its members are introduced in the following way: (a) certain "basic members" (or elements) are immediately assigned to the class; (b) a finite number of operational rules is indicated for obtaining or constructing additional members; (c) a "closure" condition is expressed, stating that only and all the members obtained in such a way are admitted in the class. A proof by induction consists then: (a) in proving that a certain property $P$ holds for the basic elements; (b) in proving that, if $P$ holds for certain members, then it also holds for the members obtained by applying the "constructive" rules to these members. In elementary arithmetic the basic element is the natural number 0 (or 1, according to preference); the constructive operation is just one, that is, the "successor" operation, and an inductive proof consists in showing that a certain property $P$ holds for 0 (or 1), and in proving that, if it holds for n, it also holds for the successor of $\mathrm{n}$. In the case of mathematical logic we can see, for example, that the class of sentences is constructed by defining as

\footnotetext{
${ }^{15}$ Poincaré has developed this view on several occasions, in the course of his often polemic discussions regarding the philosophical interpretation of mathematics. A short presentation is offered in the first chapter "On the nature of mathematical reasoning" of Poincaré (1902).
} 
basic elements the "elementary sentences", by listing a finite number of operations (depending on the sentential connectives admitted) for obtaining new "composite" sentences from already accepted sentences, by introducing a closure condition. In order to "prove by induction" that all sentences have a certain property $P$, we must first show directly that all the elementary sentences have such a property, and then prove that, if certain sentences have that property, this is preserved in the sentences obtained through the application of the admitted operational rules. Since the class of terms or the class of proofs are also defined in a constructive way, proofs by induction can by produced on the construction of a term, or on the length of a proof, and so on.

The induction of which we have spoken is more properly called "logical induction", in order to distinguish it from the (very different) induction used in the empirical sciences, and has nothing to do with the so-called "inductive logic". This is why, at the beginning of the twentieth century, certain scholars preferred to speak of a "proof by recursion" and, accordingly, one should say that such a kind of proof can be used when the referents of the discourse belong to a "recursively" defined class. Those authors had clear in mind that such a procedure was of a "logical" nature, since it denoted a particular "way of reasoning or thinking". This is evident, for example, in the title of a famous paper of Skolem, where he speaks of the "rekurrierende Denkweise" (the "recursive way of thinking", cf. Skolem (1923)).

We have devoted a rather detailed presentation to the use of the principle of logical induction because, on the one hand, it has all the features of a genuine logical principle, but, on the other hand, it is bound to the particular nature of the referents of the discourses in which it is used. Moreover, these discourses are scientific. For reasons of brevity we will not indulge in a similar detailed analysis of the two 
more examples we want to mention, also because they are well known and treated in a very abundant literature. Therefore, some hints will be sufficient.

Intuitionistic logic is known for not admitting the general validity of "classical" principles such as the excluded middle and the double negation. This logic, however, was not created "artificially" or for the sake of paradox: it was a consistent implication of the "constructivist" point of view advocated by the intuitionistic school, that deeply affected the way of conceiving the ontological status of mathematical entities, the notion of mathematical existence, and the way of conceiving the correct cognitive attitude of the mathematician.

The second well known example is quantum logic. Its development is bound to the fact that quantum "objects" are defined by means of conceptual and operational procedures that imply the impossibility in principle of simultaneously determining with a precision higher than a given threshold the value of conjugate magnitudes, with consequences regarding the purely statistical character of the predictions of the future state of a system, the outcome of experiments, etc. It has soon become clear that our knowledge of these objects could not avail itself of the traditional conceptual tools of the so-called "classical physics", and new tools had to be proposed. Some of them were theoretical principles of the new physics (such as the linear superposition, or the collapse of the wave packet); other were found in modifications of the probability calculus (using a non-kolmogorovian version of it, capable of mirroring the actual behavior of probabilities in quantum mechanics). Finally, another solution consisted in creating and formalizing a particular "logic" in which were mirrored the inferences that the scientist is obliged to adopt if he wants to adhere to the actual cognitive and ontological situation of his discourse and its referents. 


\section{WHY IS IT LOGICAL TO ADMIT SEVERAL LOGICS}

If we wanted to sketch a recapitulation of the whole of our reflections we could say that the plurality of logics is not an evidence imposed to us by the de facto situation of the existence of many logical calculi, for these are often only different "formulations" of a given logic. Such a plurality rather results from the distinction between pure and applied logic, since the plurality of logics derives from the plurality of the applications of logic, which can entail certain integrations, restrictions or modifications of the rules of pure logic. This happens because the applied logics are nothing but idealizations of the correct reasonings humans adopt (or "apply") in the different contexts of their experience, when they try to provide a justification (by means of such reasonings) of certain judgments that are not immediately supported by the kind of evidence characteristic of a given type of experience.

It is usual, when a certain theory is axiomatized, to list its "nonlogical" axioms, that is, those axioms that are meant to characterize the specificity of that theory, while the "logical" part is often reduced to the fact that a certain standardized language is used for the formulation of the axioms, and some known logical calculus is indicated as a tool for the proofs (even without explicitly listing its axioms and rules). What we are maintaining is that the "special" logics needed for making exlicit the correct reasonings adopted in a given field should receive an intermediate position: their axioms should be put beside the "nonlogical" axioms of a theory (axioms that we could call "disciplinary"), as "specific logical" axioms (the discussion presented above regarding the special "position" of the axiom of induction in elementary arithmetic exemplifies concretely what we mean here).

These logics are not "at variance" with standard alethic logic, for the simple reason that they are only more restricted "in scope", and even when they appear to contain rules or principles in contrast with those of standard logic, a careful analysis shows that such exceptions 
are only the consequence of the impossibility of finding realized (in a special context of the discourse) the conditions for the application of the standard principles and rules. Standard logic, however, remains basic in two senses. First, because it is the fremework within which all the other logics are expressed and formulated (it is, so to speak, the logic governing the metaconsiderations regarding those logics). Secondly, because even the special logics are nothing but an effort of fulfilling the task of logic as such, that is, to find explicit and controllable rules for checking the correctness of our reasonings, in the sense that they remain faithful to the links of "logical consequence". Therefore it is perfectly "logical" (in the sense that it conforms to the spirit and task of logic) to admit as many special logics as they are required for making explicit the conditions of "logical consequence" in the different types of discourse.

\section{REFERENCES}

AGAZZI, E. Modern Logic. A Survey (ed.), Reidel, Dordrecht, 1981.

—. Probability in the Sciences (ed.), Kluwer, Dordrecht, 1988a.

—_. "Probability: A Composite Concept". 1988b. In Agazzi (1988a), p. 3-26.

BELNAP, N. D. "Modal and Relevant Logics: 1977". In Agazzi (1981), p. $131-151$.

CARNAP, R. Die logische Syntay der Sprache, 1934. Engl. trans. The Logical Syntax of Language, Routledge Kegan Paul, London, 1937.

COHEN, L. J. The Implications of Induction, Methuen, London, 1970.

—. The Probable and the Provable, Oxford Univ. Press, Oxford, 1977. 
—_. "Inductive Logic 1945-1977". In Agazzi (1981), p. 353-375.

HAAK, S. Philosophy of Logic, Cambridge Univ. Press, Cambridge, 1978.

KANT, I. Anthropologie in pragmatischer Hinsicht. Frankfurt am Main: Suhrkamp, Band XII, 1977.

- Gesammelte Schriften. Editado pela Preussische. Akademie der Wissenschaften $<\mathrm{Ak}>$, Berlin, 1902.

KLEIN, F. Vergleichende Betrachtungen über neuere geometrische Forschungen, Deichert, Erlangen, 1872.

LUKASIEWICZ, J. Aristotles's Syllogistic from the Standpoint of Modern Formal Logic, Oxford Univ. Press, Oxford, 2nd edition, 1957.

POINCARÉ, H. La science et l'hypothèse, Flammarion, Paris, 1902.

PRANTL, C. Geschichte der Logik im Abendlande, Leipzig, 4. Vols, $1855-1870$.

SKOLEM, T. "Begründung der elementaren Arithmetik durch die rekurrierende Denkweise ohne Anwendung scheinbarer Veränderlichen mit unendlichem Ausdehnungsbereich", Skrifter utgit av Videnskapsselkapet i Kristiania, I. Mathematisk-videnskabelig klasse, n. 6, p. 1-38, 1923.

VERNEAUX, R. Epistémologie générale, Beauchesne, Paris, 1959.

VON WRIGHT, G. H. An Essay on Modal Logic, North Holland, Amsterdam, 1951.

—_. "Problems and Prospects of Deontic Logic. A Survey" In Agazzi (1981), p. 399-423. 